\title{
Cluster Based Random Node Mobility Model by Using K-Means Algorithm in Mobile Ad Hoc Network
}

\author{
Saba Khan ${ }^{1}$, Priya Pathak ${ }^{2}$ \\ ${ }^{1}$ Department of Computer Science GICTS RGPV, Gwalior, Madhya Pradesh, India \\ ${ }^{2}$ Department of Computer Science GICTS, RGPV, Gwalior, Madhya Pradesh, India
}

\begin{abstract}
: with the advanced technology of wireless network, the nodes interact with each other without any fixed infrastructure. This network is fully decentralized in nature and make it more efficient network to establish all over the field. There is no need to maintain wired infrastructure and easy to maintain. Nodes are free to communicate and can move at any direction without taking others opinion. In this paper, we introduced mobility models for the mobility pattern of the nodes and then the k-means cluster. Most important issue is node movement as it becomes easy to forward the data. In the proposed work, we apply cluster based random node mobility model in which nodes move freely anywhere and then create clusters to eliminate communication breaking. So in our results, we improve the network performance by increasing the throughput and packet delivery ratio.
\end{abstract}

Keywords: MANET, mobility models, K-means clustering, MATLAB tool

\section{Introduction}

Mobile Ad hoc Networks (MANET) is a type of Wireless Ad Hoc Networks (WANET) [1] where every device is independent to travel in any way. It is a self-aligning and structure-less network pointing to support movement of devices. Each device modifies its connections to further devices often resulting in a very dynamic and self-configuring topology. Each device acts as the member or router of the network.

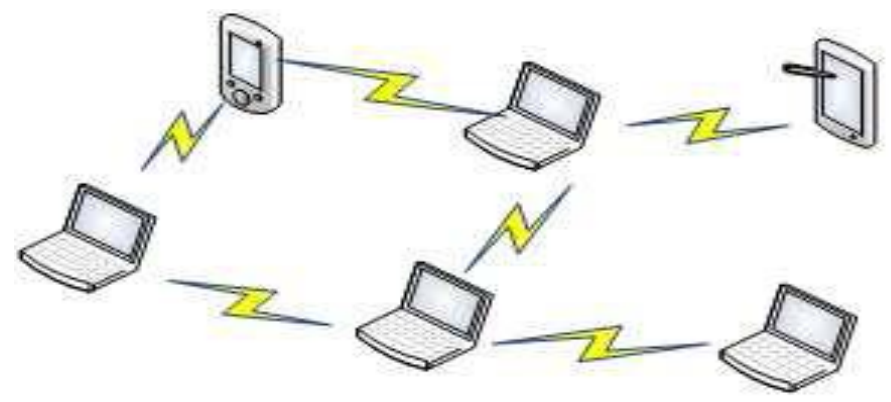

Fig .1 MANET

\section{Mobility Models}

These can be distinguished along with temporal and spatial dependencies.

a) Spatial dependency: This is a computation of how two nodes are dependent in their movement. If nodes are traveling in equal direction formerly they will have great spatial dependency.

b) Temporal dependency: This is a computation of how current velocity (direction and magnitude) are associated to preceding velocity. Nodes have equal velocity with high temporal dependency.

Given below are the descriptions of four mobility models and detailed explanation for how they emulate real world scenario. Each explanation is finished by a Network Animator (NAM) Screenshot to give a visual representation of node movement in the model. NAM is a graphic simulation display tool. It has a GUI same as a DVD player (play, rewind, fast forward, pause and so on), and also has a display speed controller.

\section{A. Random Waypoint (RWP)}

This is a generally utilized model in research group. At every moment, a node randomly selects a target and travels towards it by a velocity selected randomly from the uniform distribution [0,V_max], where $\mathrm{V} \_m a x$ is a high acceptable velocity for each node. Afterward reaching the target, the node stops for a moment specified by 'pause time' parameter. After this, it once more selects a random target and replicates the entire procedure until the termination of simulation. 


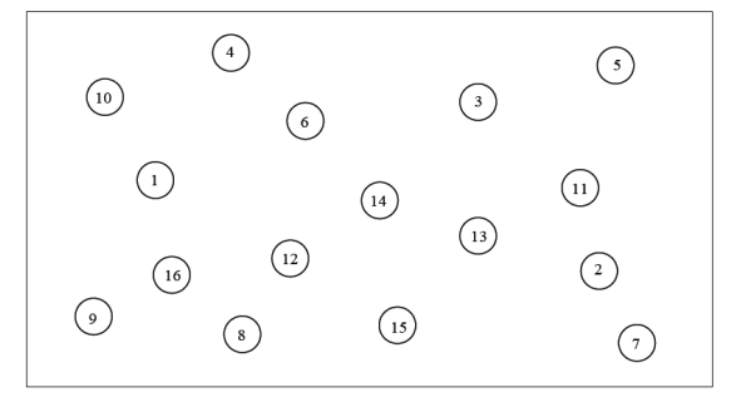

Fig 2. Topography displays the nodes movement for RWP

\section{B. Random Point Group Mobility (RPGM)}

It has an application in military battleground communication. Here every group has a logical midpoint (group leader) which defines the group's movement. Primarily each group member is equally spread in the region of group leader. Subsequently, each node has direction and velocity which is resulting by randomly differing from the midpoint. Following example of topography illustrates the nodes movement for RPGM model. The figure includes sixteen nodes using Node 9 and Node 1 like group leaders.

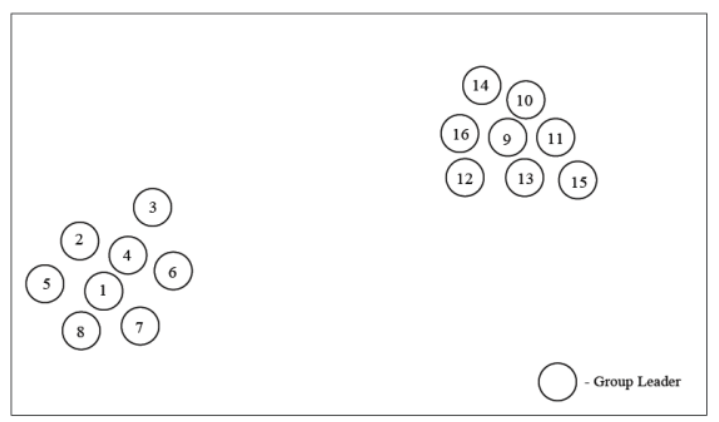

Fig 3. Diagram displaying the nodes movement in RPGM

\section{Freeway Mobility Model (FM)}

This model follows the movement behavior of nodes on a freeway. It may be utilized in replacing traffic position or a vehicle tracking on a freeway. Each node is limited to its track on the freeway. The node speed is temporally based on its preceding speed.

Following is example of topography presenting the nodes movement for FM Model [2].

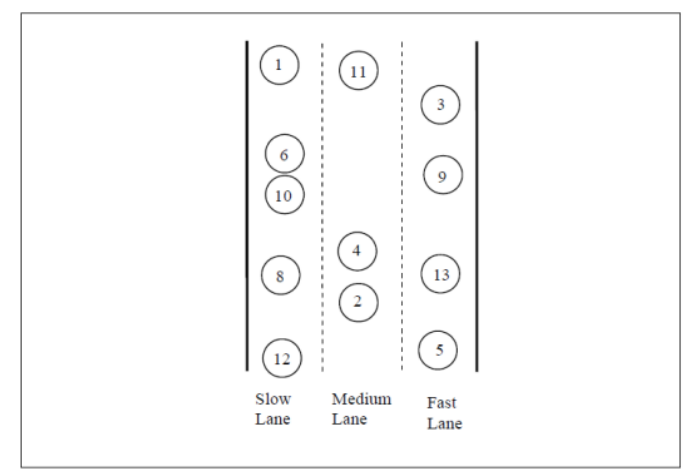

Fig 4. Topography displaying the nodes movement for FM mode

\section{Literature Survey}

Mohsin Ur Rahman, et al. [2016] evaluated the effects of various networking perspectives on the operation of mobility models. They selected Gauss-Markov (GM) and RWP models from initial group and Nomadic Community (NCMM) and RPGM models from second group. They achieved wide simulations by Network Simulator (NS-2). Simulation outcomes show that there is a drop in cost when the speed increased of MNs. In addition, node density differently impacted the presentation of both types of models [3].

Chen Chen et al. [2015] study the self-similarity characteristic of MANET traffics through simulations and then construct a fuzzy logic controlled mobility model according to traffic feature to optimize the network 
performance. First, depending on the created traffics by OPNET, the self-similarity of MANET traffics has been verified with a qualitative analysis [4].

Ahmad Foroozani, et al. [20-14] proposed a mobility mode based on the human mobility information gathered for over 6000 hours. This model captures human mobility features by presenting hotspot zones with a chart of hotspot zones as the input zone map, separating day time to several periods and modeling numerous speeds in variour places and times. Also, it models some other essential human mobility features which had been showed in preceding works. To calculate the presentation of projected model, it is matched with original collected information [5].

Ebenezer J. et al. [2014] offer a mobility model which enables realistic and easy placement of obstacles. The tragedy region is distributed into minor cells to model the obstacles in an accurate way. As there are no mobility traces present for the tragedy situations, we generate synthetic traces and compare them with the existing mobility models for tragedy scenarios. We detected important changes in the mobility parameters. This may be cause from the dynamic path selection in this model [6].

Yong Li, et al. [2012] mobility model is very essential for MANET algorithm performance evaluating. A mobility model which allocates destination for each mobile node and allows stochastic factor existing in the procedure of approaching destination is proposed. This model is used to evaluating the clustering overhead of MANET. Simulating results indicated that cluster routing is acceptable for application [7].

Pankaj Joshi, et al. [2012] three kinds of mobility node are engaged in concern destination and source node mobility and last is entire network nodes mobility. For these mobility matters both proactive mode and reactive mode of packet transformation are engaged for match, based on this matching, result is that which packet transformation technique is work better on operation mode [8].

Dharam Vir, [2012] et al. This paper provides the comparative analysis of various routing protocols used in day to day scenario under the effect of various mobility models: viz File Group and RWP Model. Results show significant effect of models on performance of routing protocols. [9]

G. R. Vijayavani, et al. [2012] a realistic model has been established by providing the real-time statement of nodes motion in an accurate health care atmosphere as an input to the simulation. The goal is to define the performance like delay, throughput, protocol overhead, and packet delivery ratio, which are generally utilized in MANET for estimating ad-hoc routing protocols- DSDV and AODV with realistic mobility model. The protocols within realistic mobility model offer high Throughput, small Normalized Routing Overhead \& Packet Delivery Ratio and Average Delay [10].

\section{K-Means}

On this section, we in short depict the direct $\mathrm{K}$ - means algorithm. The quantity of clusters $\mathrm{K}$ is thought to be settled in $\mathrm{k}$ - means clustering. Let the $\mathrm{k}$ models $(\mathrm{w} 1, \ldots$, wk $)$ be introduced to one of the $\mathrm{n}$ input designs $\left(\mathrm{i}_{1}, \ldots, \mathrm{i}_{\mathrm{n}}\right)$ for that reason,

$$
\mathrm{W}_{\mathrm{j}}=\mathrm{i}_{1}, \mathrm{j} \in\{1, \ldots, \mathrm{k}\}, 1 \in\{1, \ldots, n\}
$$

Determine 1 shows a high level description of the direct K- means clustering algorithm. $C_{j}$ is the $J_{t h}$ cluster whose regard is a disjoint subset of input examples. The quality of the clustering will depend on the next error perform [11]:

$$
\mathrm{E}=\sum_{j=1}^{k} \sum_{i_{1 \in C_{j}}}\left|i_{1}-w_{j}\right|^{2}
$$

Without problems speaking it is an algorithm to categorise or to team your objects established on attributes/points into $\mathrm{k}$ number of team. $\mathrm{K}$ is positive integer quantity. The gathering is done by means of minimizing the whole of squares of separations amongst data and the comparing cluster centroid. Hence, the intent of K-mean clustering is to classify the info.

The test now's to check which medicines have a place with cluster 1 and which medicines have a place with the other cluster. Every medicine represent to one point with two segments facilitate. Numerical illustration (handbook calculation) the elemental step of $\mathrm{K}$ - means clustering is simple. Within the commencing, we verify quantity of cluster $\mathrm{K}$ and we anticipate the centroid or middle of those clusters. We are able to take any random objects as the initial centroids or the primary $\mathrm{K}$ means may additionally function the initial centroids.

Then the $\mathrm{K}$-means algorithm will do the three steps beneath unless convergence.

Iterate until stable (=no object move group):

1.Determine the centroid coordinate

2.Examine the separation of every object the centroids

3.Team the article set up on least separation (in finding the closest centroid)

Table 1. K -means algorithm will do the three steps beneath unless convergence 


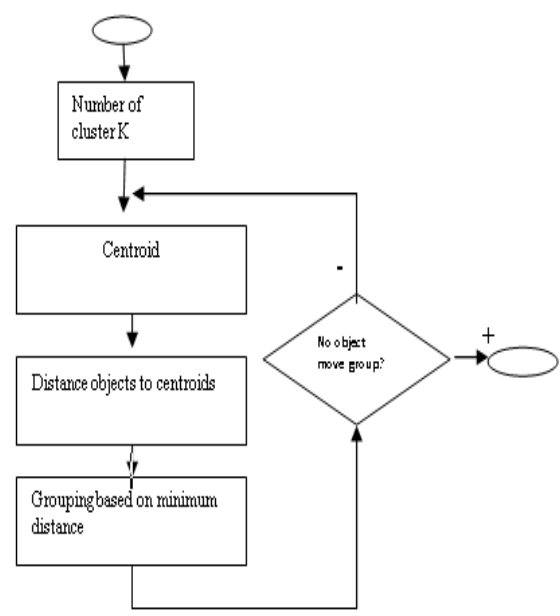

Figure 1.1 the numerical example below is given to understand this simple iteration

There are a number of functions of the K-mean clustering, range from unsupervised studying of neural network, Pattern recognitions, Classification evaluation, Artificial intelligent, image processing, machine vision, and many others. In principle, you have a couple of objects and each and every object has a few attributes and you want to classify the objects established on the attributes, then which you could observe this algorithm. Much like other algorithm, K-mean clustering has many weaknesses: when the numbers of data will not be so many, preliminary grouping will verify the cluster vastly. The quantity of cluster $\mathrm{K}$ has to be determined earlier. We never comprehend the actual cluster, using the equal data, we via no means understand which attribute contributes more to the grouping procedure considering we count on that every attribute has the identical weight. One approach to overcome these weaknesses is to make use of $\mathrm{K}$-mean clustering provided that there are on hand many data [12].

\section{Propose Work}

Node placement is one of the most important issues of network because if we know movement of node within an area packet forwarding becomes easy. There are numbers of mobility models by using these models in our network we enhance the network performance, but all have few issues with moving nature of nodes now we propose cluster based random node mobility model in which nodes freely move anywhere in network area after that when node move they create clusters so that communication break does not happen.

Algorithm:

Step1: input(number of nodes, $\mathrm{x}, \mathrm{y})$

Step2: The Random Waypoint $\left(\mathrm{V}_{\min }, \mathrm{V}_{\max }, \mathrm{T}_{\text {wait }}\right)$-Model

$>$ All participants start with random position $(\mathrm{x}, \mathrm{y})$ in $[0,1] \mathrm{x}[0,1]$

$>$ For all participants $\mathrm{i} \in\{1, \ldots, \mathrm{n}\}$ repeat forever:

- Uniformly choose next position $\left(\mathrm{x}^{\prime}, \mathrm{y}^{\prime}\right)$ in $[0,1] \mathrm{x}[0,1]$

- Uniformly choose speed $\mathrm{v}_{\mathrm{i}}$ from $\left(\mathrm{V}_{\min }, \mathrm{V}_{\max }\right]$

- $\quad$ Go from $(\mathrm{x}, \mathrm{y})$ to $\left(\mathrm{x}^{\prime}, \mathrm{y}^{\prime}\right)$ with speed $\mathrm{v}_{\mathrm{i}}$

- Wait at $\left(\mathrm{x}^{\prime}, \mathrm{y}^{\prime}\right)$ for time $\mathrm{T}_{\text {wait }}$.

- $(\mathrm{x}, \mathrm{y}) \leftarrow\left(\mathrm{x}^{\prime}, \mathrm{y}^{\prime}\right)$

Step3: The probability density function of speed of each node is then for

Step4: given by

$$
V_{\min } \leq v \leq V_{\max }
$$

Step5: since $\mathrm{f}_{\mathrm{V}}(\mathrm{v})$ is constant and

$$
f_{V}(v)=\frac{1}{V_{\max }-V_{\min }}
$$

$$
\int_{v=V_{\min }}^{V_{\max }} f_{V}(v) d v=1
$$

Step6: The Probability Density Function (pdf) of travel distance R:

$$
f_{R}(r)=\frac{2 r}{R_{\max }^{2}}
$$

$$
\text { for } 0 \leq r \leq R_{\max }
$$


Step7: The Probability Density Function (pdf) of travel time

$$
f_{S}(s)= \begin{cases}\frac{2 s}{3 R_{\max }^{2}}\left(V_{\max }^{2}+V_{\min }^{2}+V_{\max } V_{\min }\right), & 0 \leq s \leq \frac{R_{\max }}{R_{\min }} \\ \frac{2 R_{\max }}{3\left(V_{\max }-V_{\min }\right)} \frac{1}{s^{2}}-\frac{2 V_{\min }^{3}}{3 R_{\max }^{2}\left(V_{\max }-V_{\min }\right)} \cdot s, & 0 \leq \frac{R_{\max }}{R_{\min }} \leq s \leq \frac{R_{\max }}{R_{\min }} \\ 0 & s \geq \frac{R_{\max }}{R_{\min }} .\end{cases}
$$

Step8: apply k-means on this above algorithm output so that form better cluster form and new moving scenario Step9: exit

Simulation and result

This work implemented on MATLAB tool for nodes deployment.

\begin{tabular}{|l|l|}
\hline MATLAB & 2014 \\
\hline Number of nodes & 40 \\
\hline Time & $500 \mathrm{~ms}$ \\
\hline
\end{tabular}
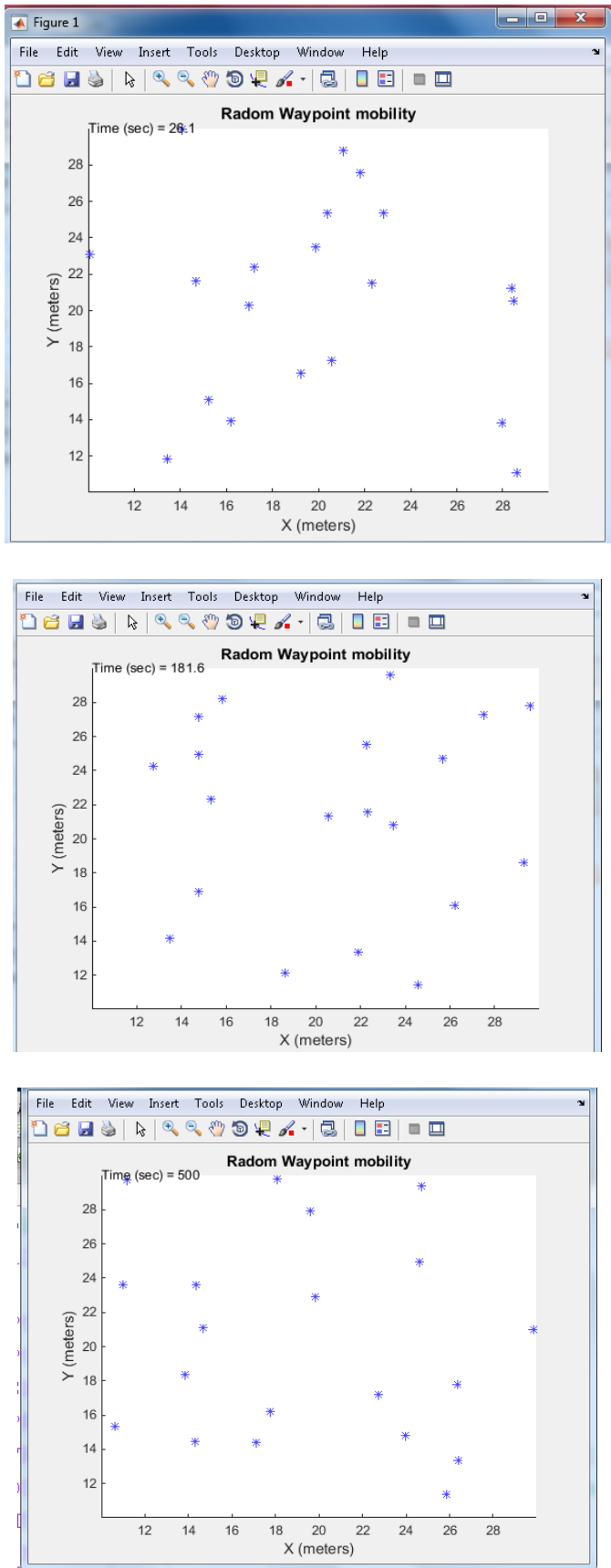
Throuhput

The total number of packets sent at a particular time is known as throughput. The figure represents that the throughput of the proposed approach is better than the existing approach.

Throughput $(\mathrm{kbps})=($ Receive size/(stop time - start time $) * 1 / 60$

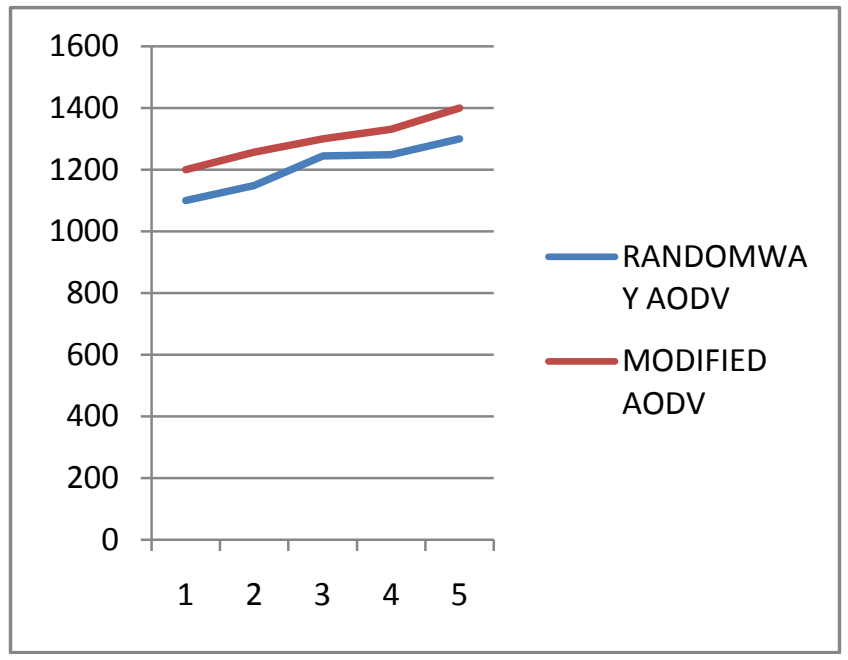

Packet Delivery Ratio

It define as the fraction of packets deliver from source toward destination. The figure represents that a PDR graph among base approach as well as proposed approach. This PDR value is better in proposed than an existing approach.

Packet Delivery Ratio $=$ Number of packet received $/$ number of packets sent

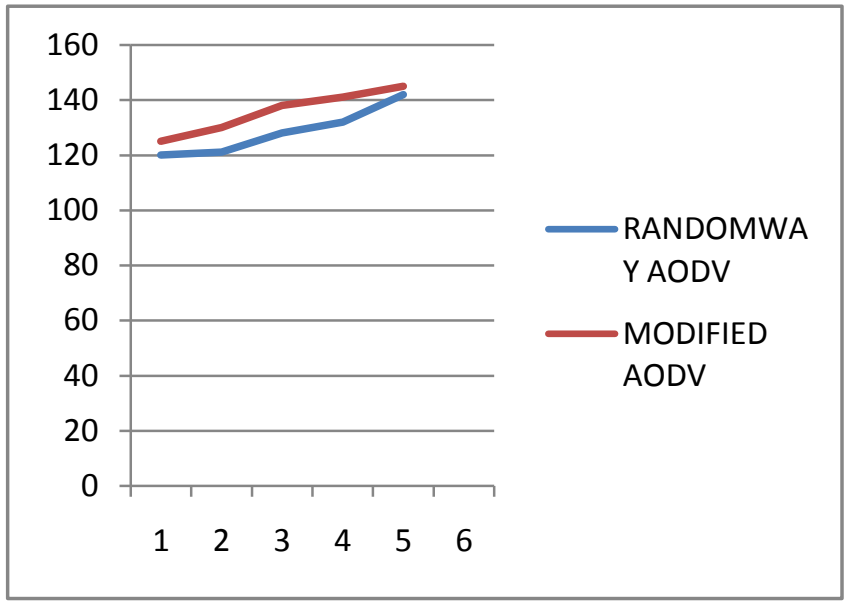

\section{Conclusion}

Mobile ad hoc network is an ad hoc network and the mobility of nodes is considered appropriately for efficient data forwarding. The movement of the nodes is very fast and it is difficult to locate the nodes to communicate directly. So in this we explain various models like RWP, RPGM and FM where the mobility of nodes mentioned. In this paper, we also explain k-means clustering to form various clusters in the network and it also reduce the communication breakage. We proposed modified AODV which is quite better than the randomway AODV. We improved the network performance which can be shown from the graphs.

\section{References}

[1] Parul Gupta “ A Literature Survey of MANET” International Research Journal of Engineering and Technology (IRJET) e-ISSN: 2395 -0056 Volume: 03 Issue: 02 | Feb-2016 www.irjet.net p-ISSN: 2395-0072

[2] Bhavyesh Divecha, Ajith Abraham, Crina Grosan and Sugata Sanyal "Impact of Node Mobility on MANET Routing Protocols Models"2012

[3] Mohsin Ur Rahman_, Aftab Alamy, and Sohail Abbasx "Investigating the Impacts of Entity and Group Mobility Models in MANETs" 978-1-5090-1252-7/16/\$31.00 c 2016 IEEE 
[4] Chen Chen and Qingqi Pei, Xi'an, P.R.China “A Fuzzy Logic Controlled Mobility Model Based on Simulated Traffics” Characteristics in MANET" 978-1-4673-7813-0/15/\$31.00 @2015 IEEE.

[5] Ahmad Foroozani, Mohammed Gharib, Ali Mohammad Afshin Hemmatyar "A Novel Human Mobility Model for MANETs Based on Real Data” 978-1-4799-3572-7/14/\$31.00 @2014 IEEE.

[6] Ebenezer J “A Mobility Model for MANET in large Scale Disaster Scenarios” 2014 17th International Conference on Computer and Information Technology (ICCIT)

[7] Yong Li, Liuyang Zhao, Hao Wang “A Novel mobility model for Clustered MANET” 978-1-61284-683-5/12/\$31.00 @2012 IEEE.

[8] Pankaj Joshi, Ajay Kumar Gautam\#, Anshul Chaudhry\#, Pankaj Punia\#, Neetu Bani "Impact of Various Mobility Model andJudgment for Selecting Mode of Network inDifferent Mobility Situation for Mobile Ad-Hoc Network (MANET)" 2012 1st International Conference on Emerging Technology Trends in Electronics, Communication and Networking

[9] Dharam Vir, S. K. Agarwal2, S.A.Imam "Quantitative Analyses and Evaluation of MANET Routing Protocols in Effect of Varying Mobility Model using Qual-Net Simulator” 978-1-4673-4805-8/12/\$31.00_c 2012 IEEE

[10] G. R. Vijayavani, ** G. Prema "Performance Comparison of MANET Routing Protocols with Mobility Model derived based on Realistic mobility pattern of Mobile Nodes" 2012 IEEE Intemational Conference on Advanced Communication Control and Computing Technologies (ICACCCT

[11] Khaled Alsabti, Sanjay Ranka and Vineet Singh “An Efficient K-Means Clustering Algorithm”2010

[12] Kardi Teknomo "K-Means Clustering Tutorial”2011. 